\title{
Assessment of Mitral Regurgitation Severity by measuring of Vena Contracta Area Using Transesophageal and Transthoracic Three-Dimensional Echocardiography
}

\author{
Ahmad S. El Garhi ${ }^{1, *}$ MSc.; Ali M. Abdalmagid ${ }^{1}$, MD; Muhammed U. Taha ${ }^{2}$, MD; Ahmed A. Mahdy ${ }^{1}$, MD
}

*Corresponding Author:

Ahmad S. El Garhi

srour_99@hotmail.com

Received for publication July 9, 2020; Accepted October 16, 2020; Published online October 16, 2020.

Copyright 2020 The Authors published by Al-Azhar University, Faculty of Medicine, Cairo, Egypt. All rights reserved. This an openaccess article distributed under the legal terms, where it is permissible to download and share the work provided it is properly cited. The work cannot be changed in anyway or used commercially.

doi: 10.21608/aimj.2020.28637.1204 ${ }^{1}$ Cardiovascular Medicine Department, Faculty of Medicine, Al-Azhar University, Cairo, Egypt.

${ }^{2}$ Cardiovascular Medicine Department, National Heart Institute, Cairo, Egypt.

\begin{abstract}
Background: Mitral regurgitation (MR) is granted the commonest native valves diseases and increases in prevalence with age. We aimed to compare (VCA) vena contracta area measurements by 3-dimensional transesophageal (3D-TEE) versus transthoracic echocardiography (3DTTE) to estimate mitral regurgitation severity.

Aim of work: to compare the accuracy of vena contracta area measurement by 3DTEE versus 3DTTE to assess the severity of mitral regurgitation.

Patient and Methods: This study was run on 40 patient attending to AlHussein University and National heart institute during the time of the study who had primary MR diagnosed by 2-dimensional transthoracic echocardiography (2D TTE). Echocardiography was done for each patient to measure: VCA by 3D-TEE and 3D-TTE considering, 3dimensional area of the proximal isovelocity surface (3D PISA) as a gold standard reference for MR severity.

Results: A comparison between 3D PISA-TEE in the grading of MR, 25 patient had severe MR, 8 patient had moderate, and 7 patient had moderate to severe MR versus 3D TTE VCA, 23 patient had severe MR, 15 patient had moderate MR and only two patient had moderate to severe MR (P-value $=0.116)$. While versus the 3D TEE VCA, 23 had severe MR, 17 patient had moderate and moderate to severe MR (P-value = 0.024*).

Conclusion: Assessment of MR grading by measuring VCAusing the 3D TEE method compared to the 3D TTE method showed that no much difference, considering 3D TEE PISA as a gold standard reference.
\end{abstract}

Keywords: echocardiography; 3-dimensional (3D); mitral regurgitation; vena contracta.

Disclosure: The authors have no financial interest to declare in relation to the content of this article. The Article Processing Charge was paid for by the authors.

Authorship: All authors have a substantial contribution to the article.

\section{INTRODUCTION}

The accurate amplitude of MR grading is extremely essential for judgement noting predicting risk and surgery. Ongoing guidelines submit the mixing of supportive, specific, and quantitative echocardiographic traits to grade the severity of MR. this including RF, vena contracta width (VCW), Rvol,and EROA is usually advocated on patient with quite mild MR whereas these quantitative manners are detailed, there's also considerable inter-observer differences between the centers. ${ }^{1}$

In the circumstance that impedes transthoracic echocardiography (TTE) (eg, acoustic shadowing from annular calcium or a mitral prosthesis), TEE shows an unobstructed view. TEE provides enhanced views of the bicuspid valve, which now includes three-dimensional (3D) reconstruction to supply detailed information on the character of the underlying pathology. It also lends information that's adjunctive to the TTE in detecting the severity. ${ }^{2}$

In the case of patient with primary MR not diagnosed properly by non invasive maneuvers. TEE is indicated to detect the grading and, mechanism of MR.

Direct assessment of MR can be done by measurement of VCA, but, overestimation of the VC area can result when multiple oblique plans that obtained by 2D transthoracic principles especially when the jet is eccentric more than central. ${ }^{3,4}$

The latest approach of real time 3-Dimensional echocardiography (RT3DE) can clarify this problem, once we've rapidly obtained the three-dimensional flow stream, a plane isinstantly aligned with the VC. 3-dimensional transthoracic echocardiography can provide a more honorable evaluation of the regurgitant volume supported an accurate impression 
of the orifice shape than 2-dimensional echocardiography. ${ }^{5}$

Recently, a much better definition of the MR jet profile with spatial resolution can be provided by a 3D matrix-array TEE transducer compared with 3D TTE provided images. ${ }^{6}$

Our study aims to compare the accuracy of vena contracta area measurement by 3DTEE versus 3DTTE to assess the severity of mitral regurgitation.

\section{PATIENTSAND METHODS}

This study was run on 40 patient were attended to $\mathrm{Al}-$ Azhar University \& NHI (National Heart Institute ) during the time of the study who had primary MR diagnosed by 2D TTE from June 2018 to July 2019 to measure, VCA by 3DTTE \&3DTEE, considering 3DTEE PISA method as the gold standard reference for MR severity.

Allpatient with primary mitral regurgitation is included in this study. And we exclude allpatient with poor image quality for TTE, contraindications of TEE, patient with functional mitral regurgitation (eg, myocardial heart disease, and ischemic dilated cardiomyopathy), patient with congenital heart disease and patient with acute cardiac events (acute heart failure and acute coronary syndrome).

All patient had a thorough evaluation, laboratory investigations, 12 lead ECG and 2D TTE performed in all patient to measure LVEDV, LVESV, and EF \% calculated. Assessment of MV to identify possible etiology, VCW measured to identify MR severity which defined as the narrowest diameter of the proximal jet measured at or immediate vicinity of the MR orifice at the leaflet tips, VC located in the parasternal long-axis view ( PSLAX ) with color flow Doppler (CFD), PISA was measured by using CFD in the apical four chambers view with zoom mode at MV with adjustment of color scale of CFD between $30-40 \mathrm{~cm} / \mathrm{s}$ Nyquist limit and measure its Radius, Continuous wave Doppler (CWD) at MV was recorded then MR peak velocity, VTI obtained, Effective Regurgitant Orifice Area (EROA) and Regurgitant volume (Rvol) computed automatically by the machine, Rvol=EROA $X$ regurgitant VTI, while Regurgitant fraction (RF\%) $=$ Rvol $/ \mathrm{MV} \mathrm{SV}$, Mitral valve stroke Volume (MVSV) the product of cross section mitral valve annulus and VTI LV inflow.

Then 3DTTE was performed to assess MR severity by measuring VC area, firstly in image acquisition for VCA is to secure a good-quality electrocardiogram tracking for prompting, the transthoracic transducer location that brings the best 2-dimensional (2D) view of the mitral valve and the MR Jet should be the initiating point for the 3dimensional (3D) acquisition, Apical views have been used in the most studies, image acquisition and end-expiratory breath-hold is requested of the patient to eradicate potential stitching artifacts, 3D color Doppler (4 beat protocols had been used, depending on heart rhythm regularity, once the 3D color
Doppler data have been acquired, images can be cropped to depict two long-axis views of the jet, finally VC area is manually traced.

3D TEE was performed and 3D color Doppler imaging data were recruited from mid oesophageal window, MR maximum velocity and VTI was measured from CWD on regurgitant MV, all data from 3DTTE, 3DTEE was transported to an offline analysis system(QLAB version 10,4 Philips medical system) and stored,3D image data were subsequently analyzed later by the same first observer to measure $3 \mathrm{D}$ vena contracta area, 3D PISA and its derived parameters, the severity of the MR was graded based on current ASE recommendation as mild $\left(<0.2 \mathrm{~cm}^{2}\right)$ with moderate or intermediate $\left(0.2\right.$ to $\left.0.39 \mathrm{~cm}^{2}\right)$ with the ability to be classified as moderate ( 0.2 to 0.29 $\left.\mathrm{cm}^{2}\right)$ and moderate to severe $\left(0.3\right.$ to $\left.0.39 \mathrm{~cm}^{2}\right)$, or severe $\left(\geq 0.40 \mathrm{~cm}^{2}\right)$.

Statistical analysis: implemented by the SPSS statistical package version 16 . The data had beenanalyzed using (IBM Corp, Chicago, IL, USA) Qualitative data had been given in the form of numbers and percentages and quantitative variables as mean and standard deviation (SD). A comparison between quantitative variables had been carried out by the student T-test of two independent samples. Chi-square test $\left(\mathrm{X}^{2}\right)$, had been used to compare qualitative variables. 


\section{RESULTS}

Assessment of MR severity by different echocardiographic techniques showed astatistically significant difference between VCA measured by 3D-TTE compared to the 3D-TEE method with p-value $=0.005$. (Table 1 ).

\begin{tabular}{|c|c|c|c|c|c|c|c|c|c|c|}
\hline & \multicolumn{4}{|c|}{ 3D-VCA $\left(\mathbf{c m}^{2}\right)$} & \multicolumn{3}{c|}{ Differences } & \multicolumn{3}{c|}{ Paired Test } \\
\cline { 2 - 11 } & \multicolumn{3}{|c|}{ Range } & Mean & \pm & SD & Mean & SD & T & $\begin{array}{c}\text { P- } \\
\text { value }\end{array}$ \\
\hline 3D-TTE & 0.22 & - & 1.2 & 0.473 & \pm & 0.215 & -0.041 & 0.087 & -2.943 & $\begin{array}{c}0.005 \\
*\end{array}$ \\
\hline 3D-TEE & 0.27 & - & 1.7 & 0.513 & \pm & 0.270 & -0.94 & & \\
\hline
\end{tabular}

Table 1: Difference between 3D VCA range by TTE and TEE.

Also, there was a strong positive correlation between 3D PISA-EROA and corresponding VCA derived by 3D TTE with r-value $=0.459$ and r-value $=0.373$ derived by VCA-TEE. (Table 2) .

\begin{tabular}{|c|c|c|c|c|}
\hline \multirow{2}{*}{} & \multicolumn{2}{|c|}{ 3D- TTE VCA $\left(\mathbf{c m}^{2}\right)$} & \multicolumn{2}{|c|}{ 3D-TEE VCA $\left(\mathbf{c m}^{2}\right)$} \\
\cline { 2 - 5 } & $\mathbf{R}$ & P-value & $\mathbf{R}$ & P-value \\
\hline 3D-PISA EROA $\left(\mathbf{c m}^{2}\right)$ & 0.459 & $0.003^{*}$ & 0.373 & $0.018^{*}$ \\
\hline 3D-PISA R vol $(\mathbf{m l})$ & 0.262 & 0.102 & 0.189 & 0.243 \\
\hline 3D-PISA RF $\%$ & 0.235 & 0.144 & 0.206 & 0.202 \\
\hline
\end{tabular}

Table 2: Correlation between 3D PISA parameters and 3D VCA measurement.

A comparison between 3D PISA-TEE in the grading of MR, 25 patient had severe MR, 8 patient had moderate, and 7 patient had moderate to severe MR versus 3D TTE VCA, 23 patient had severe MR, 15 patient had moderate MR and only two patient had moderate to severe MR (P-value $=0.116)$. While versus the 3D TEE VCA, 23 had severe MR, 17 patient had moderate and moderate to severe MR $\left(\mathrm{P}\right.$-value $\left.=0.024^{*}\right)$. (Table 3$)$.

\begin{tabular}{|c|c|c|c|c|c|c|c|c|c|c|c|}
\hline & & \multicolumn{8}{|c|}{ 3D-PISA-grading } & \multirow{2}{*}{\multicolumn{2}{|c|}{ Chi-Square }} \\
\hline & & \multicolumn{2}{|c|}{ Moderate } & \multicolumn{2}{|c|}{ Moderate to Severe } & \multicolumn{2}{|c|}{ Severe } & \multicolumn{2}{|c|}{ Total } & & \\
\hline & & $\mathbf{N}$ & $\%$ & $\mathbf{N}$ & $\%$ & $\mathbf{N}$ & $\%$ & $\mathbf{N}$ & $\%$ & $X^{2}$ & P-value \\
\hline \multirow{2}{*}{$\begin{array}{l}\text { VCW-2D } \\
\text { MR grading }\end{array}$} & Moderate & 6 & 75.00 & 7 & 100.00 & 13 & 52.00 & 26 & 65.00 & \multirow{2}{*}{8.181} & \multirow{2}{*}{$0.017 *$} \\
\hline & Severe & 2 & 25.00 & 0 & 0.00 & 12 & 48.00 & 14 & 35.00 & & \\
\hline \multirow{3}{*}{$\begin{array}{c}\text { 3D- TTE VCA } \\
\text { MR grading }\end{array}$} & Moderate & 5 & 62.50 & 4 & 57.14 & 6 & 24.00 & 15 & 37.50 & \multirow{3}{*}{7.415} & \multirow{3}{*}{0.116} \\
\hline & Moderate to Severe & 0 & 0.00 & 1 & 14.29 & 1 & 4.00 & 2 & 5.00 & & \\
\hline & Severe & 3 & 37.50 & 2 & 28.57 & 18 & 72.00 & 23 & 57.50 & & \\
\hline \multirow{3}{*}{$\begin{array}{c}\text { 3D-TEE VCA } \\
\text { MR grading }\end{array}$} & Moderate & 5 & 62.50 & 5 & 71.43 & 4 & 16.00 & 14 & 35.00 & \multirow{3}{*}{11.251} & \multirow{3}{*}{$0.024 *$} \\
\hline & Moderate to Severe & 0 & 0.00 & 0 & 0.00 & 3 & 12.00 & 3 & 7.50 & & \\
\hline & Severe & 3 & 37.50 & 2 & 28.57 & 18 & 72.00 & 23 & 57.50 & & \\
\hline
\end{tabular}

Table (3): Relation between 3D PISA assessment methods and VCW measurements by 2D-TTE, 3D-TTE \& 3DTEE in the assessment of MR grading.

\section{DISCUSSION}

VC is feasible and less technically stringent than other techniques and may be less dependenton burdening circumstances. It's the cross-sectional area of the regurgitant jet of MR that canbe measured by( (EDTRand corresponds htiw (EROA).

In our study, there was no much difference between 3D TTE VCA and 3D TEE VCA considering 3D TEE PISA as a gold standard reference.

In our study, the degree of MR assessed by 3D TTEVCA: (23 patient) had severe, (15 patient) had moderate and only two patient had moderate to severe MR, and by 3D TEE-VCA: (23 patient) had severe MR, (14 patient) had moderate MR and only three patient had moderate to severe MR.

In our study, there was a moderately strong positive correlation between 3D PISA EROA and 3D VCA measured by TTE with (r-value $=0.459$, and $\mathrm{p}$-value $\left.=0.003^{*}\right)$, and by TEE with (r-value $=0.373$, and $\mathrm{p}$ value $=0.018^{*}$ ) in the assessment of MR severity.

In agreement with Iwakura, ${ }^{7}$ et al. who studied 106 patient with at least moderate MR showed that the 3D-VC area strongly correlated with the quantitative Doppler method. Furthermore, agreement with Little, ${ }^{8}$ et al. who studied 61 patient with at least mild MR in the assessment of 3D VC area with reference standard Doppler-derived EROA, mentioned that there was a strong correlation with Doppler-derived 
EROA and 3D VC area particularly in a patient with moderate to severe MR.

In our study, we found that the MR severity significant increase in 3D TEE PISA method compared to 2D TTE VCW with P-value $=0.017$, and 3D TEE VCA with P-value $=0.024$. while insignificant difference compared to 3D TTE VCA with P-value $=0.116$.

In agreement with Zeng, ${ }^{9}$ et al. who studied 83 patient with mild MR experienced 2D echocardiography and 3D echocardiography by whichdirect planimetry of VCA had performed, quantification of MR incorporated 2D PISA-ROA approach, VCW, and the ratio of jet area to left atrial area. They revealed that the 3D VCA contributes a single, directly visualized, and reliable measurement of ROA, which grades MR severity according to current clinical practice depending on the American Society of Echocardiography recommended 2D integrablemanner.

\section{CONCLUSION}

3D VCA provides a single, directly visualized, and credible measurement of ROA, autonomous geometric and flow assumptions, which grades MR severity. 3D VCA TTE/TEE approach is recommended in clinical practice to contribute a more accurate assessment of MR severity. Measuring of the VCA by 3D TEE/TTE methods showed that no much difference according to MR grading. Although 3D TEE provides more accurate results clearer images and provides better Doppler quality than 3D TTE method, considering 3D TEE PISA as a gold standard reference. But TEE has various barriers, The patient must follow the ASA NPO guideline. TEE needs a group of medical staff. TEE takes more time than TTE. It may be annoyed for the patient, who may require sedation or general anaesthesia and Some complications are associated with the procedure, as oesophageal perforation and adverse effects from the medication.

\section{REFERENCES}

1. Paaladinesh T, Dermot P, Patrick C, et al. Quantitative Assessment of Mitral Regurgitation. JACC Cardiovasc Imaging. 2012; 11(5): 1161-75.
2. Buck T, Mucci R, Guerrero J, Holmvang G, Handschumacher M,et al. The power velocity integral at the vena contracta: A new method for direct quantification of regurgitant volume flow. Circ. Cardiovascular Imaging. 2000;102(9):105361.

3. Bai A, Steinberg M, Showler A, Burry L, Sacha Bhatia R, et al. Diagnostic Accuracy of Transthoracic Echocardiography for Infective Endocarditis Findings Using Transesophageal Echocardiography as the Reference Standard: A Meta-Analysis. J Am Soc Echocardiogr. 2017; 30(7):639-46.

4. Enriquez M, Avierinos J, Messika D, Detaint D, Capps M, et al. Quantitative determinants of the outcome of asymptomatic mitral regurgitation. $N$ Engl J Med. 2005; 352(9): 875-83.

5. Monaghan MJ. Role of real time 3D echocardiography in evaluating the left ventricle. Heart. 2006; 92(1): 131-6.

6. Yosefy C, Hung J, Chua S, Vaturi M, Levine R, et al. Direct measurement of vena contracta area by real-time 3-dimensional echo-cardiography for assessing the severity of mitral regurgitation. $\mathrm{Am} \mathrm{J}$ Cardiol. 2009; 104(7): 978-83.

7. Iwakura K, Ito $\mathrm{H}$, Kawano S, et al. Comparison of orifice area by transthoracic three-dimensional Doppler echocardiography versus proximal isovelocity surface area (PISA) method for assessment of mitral regurgitation. Am J Cardiol. 2006; 97(11): 1630-7.

8. Little SH, Pirat B, Kumar R, et al. Threedimensional color Doppler echocardiography for direct measurement of vena contracta area in mitral regurgitation: in vitro validation and clinical experience. JACC Cardiovasc Imaging. 2008; 1(6): 695- 704.

9. Zeng X, Levine RA, Hua L, Robert A, Lanqi H, et al. Diagnostic value of vena contracta area in the quantification of mitral regurgitation severity by color Doppler 3D echocardiography. Circ. Cardiovascular Imaging. 2011; 4(5):110-9. 